\title{
The appearance of an interval of energies that contain the whole diamagnetic contribution to NMR magnetic shieldings
}

\author{
Alejandro Maldonado and Gustavo A. Aucar ${ }^{a}$ \\ Department of Physics, Northeastern University of Argentina, Av. Libertad 5500, W 3404 AAS Corrientes, \\ Argentina
}

(Received 8 May 2007; accepted 28 August 2007; published online 19 October 2007)

\begin{abstract}
Working within relativistic polarization propagator approach, it was shown in a previous article that the electronic origin of diamagnetic contributions to NMR nuclear magnetic shielding, $\sigma^{d}$, are mostly excitations that fit in a well defined interval of energies such that $2 m c^{2} \leqq\left(\varepsilon_{i}-\varepsilon_{\bar{s}}\right)<4 m c^{2}$. That interval of energies does not have, in principle, any physical reason to be so well defined, and gives a large amount of the total contribution to $\sigma^{d}$, e.g., close to $98 \%$ of it. Then a further study is given in this article, where we show some of the main characteristics of that interval of energy, such as its universal appearance and basis set independence. Our main result is the finding that $\sigma^{d}$ is completely described by that interval of excitation energies, i.e., there is no contribution arising from outside of it. Most of the contributions belonging to that interval arise from virtual electronic energies larger than $-3 m c^{2}$. For heavier atoms, there are few contributions from states with virtual negative energies smaller than $-3 m c^{2}$. The model systems under study were noble gases, $\mathrm{XH}(\mathrm{X}$ $=\mathrm{Br}$, I, and $\mathrm{At}), \mathrm{XH}_{2}(\mathrm{X}=\mathrm{O}, \mathrm{S}, \mathrm{Se}, \mathrm{Te}$, and $\mathrm{Po}), \mathrm{XH}_{3}(\mathrm{X}=\mathrm{N}, \mathrm{P}, \mathrm{As}, \mathrm{Sb}$, and $\mathrm{Bi}) ; \mathrm{XH}_{4}(\mathrm{X}=\mathrm{Sn}$ and $\mathrm{Pb})$, and $\mathrm{SnXH}_{3}(\mathrm{X}=\mathrm{Br}$ and I). The pattern of contributions of occupied molecular orbitals (MOs) is also shown, where the $1 s_{1 / 2}$ is the most important for excitations ending in the bottom half part of the above mentioned interval. On the other hand, the contribution of the other occupied MOs are more important than that of $1 s_{1 / 2}$ for the other part of such interval. We also show that $\sigma^{d}$ is electron correlation independent within both relativistic and nonrelativistic domain. In the case of $\sigma^{p}$, we find out a clear dependence of electron correlation effects with relativistic effects, which is of the order of 30\% for $\mathrm{Pb}_{\text {in }} \mathrm{PbH}_{4}$. (C) 2007 American Institute of Physics. [DOI: 10.1063/1.2787003]
\end{abstract}

\section{INTRODUCTION}

The analysis of the electronic origin of diamagnetic contributions to atomic and molecular magnetic properties with full relativistic formalisms is still a matter of investigation. In such regime, the Hamiltonian does not contain the usual nonrelativistic magnetic vector potential depending term, the $\mathbf{A}^{2}$ term, because the free particle Dirac $\boldsymbol{\alpha} \cdot \mathbf{p}$ term is replaced (applying minimal coupling prescription) by $\boldsymbol{\alpha} \cdot(\mathbf{p}-e / c \mathbf{A})$ when the electronic particle is in an external magnetic field. That means that, in this case, diamagnetism cannot be explained quantum mechanically from a term in the Hamiltonian that depends on the square of the vector potential. There were several recent proposals to shed some light on this problem, ${ }^{1-5}$ some of them looking for explanation only through the positive-energy electronic spectra. ${ }^{3,5}$

If one wants to exploit the whole physical novelty that is included in Dirac's theory, one must continue working with its full formalism. One of its greatest success, the discovery of positron particles, was predicted by the fact that the full solution of Dirac's equation has two branches of oneelectron energy states. A proper way of working with particles that have only positive-energy states and still have the full relativistic characteristics (Dirac's flavor) is to go one step further and apply the quantum electrodynamic

\footnotetext{
${ }^{a)}$ Author to whom correspondence should be addressed. Electronic mail: gaa@unne.edu.ar
}

formalism. ${ }^{6,7}$ In this last case, it was shown that diamagnetism arises from both "virtual" creation and annihilation of electron-positron pairs.

Following preliminary ideas first introduced by Sternheim $^{8}$ and then extended by Pyykkö ${ }^{9}$ to one-electron systems a few years ago, it was shown, by theory and calculations, that the large diamagnetic contribution to nuclear magnetic shieldings is related to electronic excitations from occupied electronic states to virtual negative-energy electronic states. ${ }^{1}$ It means that negative-energy states do have an unavoidable contribution to the evaluation of nuclear magnetic shieldings. When "virtual excitations" to Dirac's branch of one-electron negative-energy states are neglected from the outset, diamagnetic contributions to any magnetic atomic or molecular property do not appear. ${ }^{1,10}$

Concerning the understanding of diamagnetism within relativistic polarization propagator approach, an interesting further step was done when the pure zeroth-order approach (PZOA) was applied to rare gas atoms and $\mathrm{XH}(\mathrm{X}=\mathrm{Br}$ and $\mathrm{I})$ type of molecules. ${ }^{4}$ An unexpected and well defined interval of excitation energies (from occupied to negative-energy one-electron states) which contain almost the whole diamagnetic contribution to nuclear magnetic shieldings was found. ${ }^{4}$ That interval of excitation energies is such that $2 m c^{2} \leqq\left(\varepsilon_{i}\right.$ $\left.-\varepsilon_{\bar{s}}\right)<4 m c^{2}$. The subscript $i(\bar{s})$ means occupied (virtual negative-energy) electron states. From these findings, there are still several not yet well understood questions: (i) Is that 
behavior universal? (ii) Which is the pattern of contributions of the first occupied molecular orbital (MO) compared with the total contributions of all other occupied MOs? (iii) Is it possible to get a converged and accurate number for the percentage of the total $\sigma^{d}$ that arise from excitations belonging to such interval? One natural answer would be "close to $100 \%$." One of the aims of this article is mainly related to their answers.

There is also another still open question which we address here: Is it possible to acquire a first approach to the relationship between relativity and electron correlation effects? We will show that relativistic effects do modify the contribution of electron correlation.

The shielding of heavy atoms were analyzed in the following model molecular systems: noble gases, $\mathrm{XH}(\mathrm{X}=\mathrm{Br}, \mathrm{I}$, and $\mathrm{At}), \mathrm{XH}_{2}(\mathrm{X}=\mathrm{O}, \mathrm{S}, \mathrm{Se}, \mathrm{Te}$, and $\mathrm{Po}), \mathrm{XH}_{3}(\mathrm{X}=\mathrm{N}, \mathrm{P}, \mathrm{As}$, $\mathrm{Sb}$, and $\mathrm{Bi}), \mathrm{XH}_{4}(\mathrm{X}=\mathrm{Sn}$ and $\mathrm{Pb})$, and $\mathrm{SnXH}_{3}(\mathrm{X}=\mathrm{Br}$ and $\mathrm{I})$.

In Sec. II, a brief sketch of the relativistic polarization propagator at both levels, random phase approximation (RPA) and PZOA, is given together with the development of the principal propagator as a series. Computational details used for all calculations are given in Sec. III, and Sec. IV is devoted to a discussion of the results. We start giving the results of relativistic calculations at the RPA level of approach with both restricted and unrestricted kinetic balance (RKB and UKB) prescriptions. Then we compare calculations of $\sigma^{d}$ and $\sigma^{p}$ at RPA and PZOA levels of approach in different model compounds, and also an analysis of diamagnetic contributions with that from the series which can replace the principal propagators is done. It is shown that calculations of $\sigma^{d}$ with the series seems to reproduce $100 \%$ of the calculations at PZOA level of approach, which are very close to RPA results. The analysis of the pattern of contributions to $\sigma^{d}$ from the first occupied four-component molecular orbital and the whole interval of negative-energy electronic states is shown. Concluding remarks are presented in Sec. V.

\section{THEORY}

Within the complete relativistic polarization propagator approach (RPPA), ${ }^{10}$ it makes no sense to consider separately both kind of contributions, e.g., para- and diamagnetic, to the nuclear magnetic shielding. In fact, only the total shielding is gauge independent. Within RPPA theory, that separation arises naturally when mixed contributions (virtual positiveand virtual negative-energy electronic states) to the perturbed matrix elements are neglected. ${ }^{1}$

The NMR nuclear magnetic shielding tensor for a nucleus $K$ within RPPA formalism can be written as ${ }^{1,10}$

$$
\sigma(K)=\left\langle\left\langle\mathbf{V}_{K} ; \mathbf{V}_{B}\right\rangle\right\rangle,
$$

where the operators $\mathbf{V}_{K}$ and $\mathbf{V}_{B}$ are related to the external perturbations that intervene in the specific polarization propagator. These operators are included in the perturbative Hamiltonian

$$
H=c \boldsymbol{\alpha} \cdot\left(\mathbf{A}_{K}+\mathbf{A}_{B}\right),
$$

where

$$
\mathbf{A}_{K}=\boldsymbol{\mu}_{K} \times \frac{\mathbf{r}_{K}}{r_{K}^{3}}, \quad \mathbf{A}_{B}=(1 / 2) \mathbf{B} \times \mathbf{r}_{G},
$$

so that

$$
H=\boldsymbol{\mu}_{K} \cdot\left(\mathbf{V}_{K}+\mathbf{V}_{B}\right) .
$$

In Eq. (4),

$$
\mathbf{V}_{K}=\boldsymbol{\alpha} \times \frac{\mathbf{r}_{K}}{r_{K}^{3}}
$$

and

$$
\mathbf{V}_{B}=\frac{1}{2} \boldsymbol{\alpha} \times \mathbf{r}_{G}=\frac{1}{2} \boldsymbol{\alpha} \times\left(\mathbf{r}-\mathbf{R}_{G}\right) .
$$

$\mathbf{r}_{K}$ is the nuclear position, and the gauge origin of the external magnetic field is set at the position $\mathbf{R}_{G}$.

Equation (1) can be reexpresed so that all virtual electronic excitations to be considered are written explicitly as

$$
\sigma(K)=\sum\left\{\mathbf{V}_{K \text {;ee,ep,pp }} \mathbf{P}_{\text {ee,ep,pp }}^{-1} \mathbf{V}_{B ; \text { ee,ep,pp }}\right\} .
$$

In this equation ee, ep, and pp means that only excitations to positive-energy electronic states, excitations to a mixing of positive-energy and negative-energy electronic states, and excitations only to negative-energy electronic states, are included, respectively. The operator $\mathbf{P}^{-1}$ is called the principal propagator and gives the contribution of the unperturbed electronic pathways that are involved in the transmission of both interactions considered for nuclear magnetic shieldings, i.e., $\mathbf{V}_{K}$ and $\mathbf{V}_{B}{ }^{11}$

It was shown that the ep part gives quite small contributions to $\sigma .{ }^{1}$ In fact, it gives contributions of order $\mathrm{O}\left(c^{-1}\right)$ compared with contributions of order $\mathrm{O}\left(c^{0}\right)$ arising from the other parts of $\mathbf{P}^{-1}$. Hence, as just mentioned above, neglecting all ep contributions, Eq. (7) can be divided into two independent parts: the ee and pp parts. The $\sigma^{d(p)}$ term is obtained when considering only pp (ee) contributions:

$$
\sigma^{d(p)}(K)=\sum\left\{\mathbf{V}_{K ; \mathrm{pp}(\mathrm{ee})} \mathbf{P}_{\mathrm{pp}(\mathrm{ee})}^{-1} \mathbf{V}_{B ; \mathrm{pp}(\mathrm{ee})}\right\} .
$$

The principal propagator is a supermatrix that contains terms, at the RPA level of approach, that depend on the inverse of the difference between occupied and virtual excited electronic molecular states and terms containing twoelectron integrals. Neglecting these last terms, the PZOA is obtained. So, at PZOA level of approach, the diamagnetic term is

$$
\sigma^{d}(K)=\sum_{i \bar{s}}\left\{\mathbf{V}_{K ; i \bar{S}} \mathbf{P}_{i \bar{s} ; i \bar{s}}^{-1} \mathbf{V}_{B ; i \bar{s}}\right\}+\text { c.c. },
$$

where $i(\bar{s})$ stand for occupied (virtual negative-energy) electronic states, and the second term of the right hand side of Eq. (9) is the complex conjugate. The contribution of each occupied MO at this level of approach is obtained from

$$
\sigma_{i}^{d}=\sum_{\bar{s}}\left\{\mathbf{V}_{K ; i \bar{s}} \frac{1}{\varepsilon_{i}-\varepsilon_{\bar{s}}} \mathbf{V}_{B ; i \bar{s}}\right\}+\text { c.c. } .
$$
as $^{4}$ 


$$
\mathbf{P}_{i \bar{s} ; \bar{s}}^{-1}(\Delta)=\left(\varepsilon_{i}-\varepsilon_{\bar{S}}\right)^{-1}=\frac{1}{2 m c^{2}} \frac{1}{1+\Delta},
$$

where

$$
\Delta=\frac{\left(\varepsilon_{i}-\varepsilon_{\bar{s}}\right)-2 m c^{2}}{2 m c^{2}} .
$$

In Eq. (11), $\Delta$ is positive and can have any allowed value consistent with the eigenvalues of Dirac-Hartree-Fock (DHF) solutions. If one considers only contributions with $\Delta$ less than 1, the last term of Eq. (11) can be expressed as a series

$$
\mathbf{P}_{i \bar{s} ; i \bar{s}}^{-1}(\Delta)=\sum_{q=0}^{n} S_{q}(\Delta)=\sum_{q=0}^{n} \frac{1}{2 m c^{2}}(-\Delta)^{q} .
$$

Considering only the first two terms of this series, i.e., $n=1$, one gets exactly those terms used in the linear response-elimination of small component (LR-ESC) formalism ${ }^{12-14}$ or the equivalent Breit-Pauli perturbational approach. ${ }^{15,16}$ If one sums up all terms of the series of Eq. (13), converged results may or may not be coincident with PZOA results. Here, it must be stressed that the series of Eq. (13) strictly converges only for $\Delta$ less than 1 .

\section{COMPUTATIONAL DETAILS}

All calculations of NMR- $\sigma$ at RPA level of approach and the matrix elements of operators $\mathbf{V}_{K}$ and $\mathbf{V}_{B}$ [see Eq. (1)] were done with DIRAC program package. ${ }^{17}$ PZOA values of $\sigma$ were obtained from a slight modification of DIRAC. One should realize that there is a large saving of computational effort when the calculations of $\sigma^{d}$ are done at PZOA level or with the series of Eq. (13). The time saved is more than 50\% of that needed for RPA calculations. This is due to the fact that, at this level, we do not need to work with the property module of the DIRAC code.

For nonrelativistic calculations, the speed of light was taken as $10 c(c=137.0359998$ a.u. $)$. Experimental geometries were used for all molecular models and taken from Refs. 18 and 19. The gauge origin was assigned to the position of the heavy atom unless otherwise specified, and the Gaussian nuclear model was used in all calculations.

Both RKB and UKB prescriptions were used to generate small components from large components for the fourcomponent basis set. ${ }^{1,20}$ Sadlej basis set $^{21}$ was chosen in most cases, with several tighter and more difuse Gaussian functions included to get converged results. The scheme for including more Gaussian functions was the usual: (i) tight basis functions were added to $s$ and $p$ blocks with exponents related as $\alpha_{i+1} / \alpha_{i}=3$ from the largest exponent of the block; (ii) diffuse basis functions were added to the $d$ and $f$ blocks with the same relation between exponents as for tight basis functions. In the case of $\mathrm{Se}$ and $\mathrm{Te}$, the basis set used was taken from Ref. 1. Faegri's basis sets were used for As, Bi, $\mathrm{Sn}, \mathrm{Pb}$, and noble gases, ${ }^{22}$ with tighter and more difuse basis functions added, following the scheme mentioned above.

\section{RESULTS AND DISCUSSION}

Within RPPA theory, the electronic origin of diamagnetic contributions to $\sigma$ is more easily analyzed when working at PZOA level of approach. At this level, two-electron integrals are not considered in each element of the principal propagator [see Eq. (8)]. At RPA level, the principal propagator matrices are built up from two matrices: (i) the one in which matrix elements contain terms where the average value of the unperturbed Hamiltonian is taken between two singleexcited states, and (ii) another matrix in which matrix elements of the Hamiltonian are taken between the reference state and the double-excited states. ${ }^{23}$ So when doing RPA calculations, the orbital relaxation due to the external magnetic field is included. Their corresponding correction terms are coincident with that of including electronic correlation at single-excited configuration interaction and the matrix elements of the Hamiltonian between double-excited states and reference state. ${ }^{23}$ These considerations are valid with independence of the domain within which calculations are done, e.g., relativistic or nonrelativistic.

Comparative results for calculations with medium-size basis set using RKB and UKB prescriptions are given in Table I. It is shown that one gets better results with a smaller basis set when using UKB prescriptions. All the results we present here are independent of the actual prescription (RKB or UKB) used; then for practical reasons which are mainly related to computational requirements, we will continue our analysis by working only with RKB.

\section{A. Electron correlation dependence on relativity for nuclear magnetic shieldings}

Previous nonrelativistic (NR) calculations of $\sigma$ with polarization propagators at different levels of approach have shown that much of the electron correlation is just included at RPA level of approach for model compounds such as those studied here. For example, $\sigma^{\mathrm{SOPPA}}(\mathrm{O})\left[\sigma^{\mathrm{RPA}}(\mathrm{O})\right]$ $=330.62 \mathrm{ppm} \quad(324.72 \mathrm{ppm})$ in $\mathrm{H}_{2} \mathrm{O}$, and $\sigma^{\mathrm{SOPPA}}(\mathrm{N})\left[\sigma^{\mathrm{RPA}}(\mathrm{N})\right]=266.04 \mathrm{ppm}(260.61 \mathrm{ppm})$ in $\mathrm{NH}_{3} \cdot{ }^{24}$ A similar behavior was observed for $\mathrm{PH}_{3}$ and $\mathrm{H}_{2} \mathrm{~S}$ model compounds. ${ }^{25}$ These studies also show that the paramagnetic term calculated at RPA level is within 5\% of the difference when compared with calculations at SOPPA level: For $\mathrm{H}_{2} \mathrm{O}$, $\sigma^{p, \mathrm{SOPPA}}(\mathrm{O})\left[\sigma^{p, \mathrm{RPA}}(\mathrm{O})\right]=-84.45 \mathrm{ppm}(-90.61 \mathrm{ppm}), \quad$ and $\sigma^{p, \mathrm{SOPPA}}(\mathrm{N})\left[\sigma^{p, \mathrm{RPA}}(\mathrm{N})\right]=-87.96 \mathrm{ppm}(-93.54 \mathrm{ppm}) \quad$ for $\mathrm{NH}_{3}$. Then we may argue that calculations of $\sigma^{p}(\mathrm{X})$ at RPA level within the relativistic domain will contain most of the electron correlation effects, assuming that relativity would hardly modify the pattern of correlation contributions at each level of approach: PZOA, RPA, and SOPPA.

In a previous work, it was shown that calculations of $\sigma^{d}$ at PZOA level of approach are quite close to their equivalent calculations at RPA level for rare gases and halogen hydrides. ${ }^{4}$ An extension to another model molecular system is then necessary. In Table II, it is shown that diamagnetic contributions to $\sigma$ at both levels of approach give almost exactly the same results even for the heaviest elements of each series. Within both regimes, relativistic (R) and NR, the 
TABLE I. Comparative results between calculations using RKB and UKB prescriptions, with medium-size converged basis sets. All calculations were done at RPA level and are given in ppm.

\begin{tabular}{|c|c|c|c|c|c|}
\hline & $\sigma^{p}$ & $\sigma^{d}$ & $\sigma$ & $\sigma$ (Others) & Expt. \\
\hline \multicolumn{6}{|c|}{$\mathrm{XH}_{2}$} \\
\hline $\mathrm{O}(\mathrm{RKB} / 15 s 10 p 6 d 1 f)$ & -82.18 & 388.38 & 306.20 & $340.34,{ }^{\mathrm{a}} 329.7^{\mathrm{b}}$ & \\
\hline$(\mathrm{UKB} / 14 s 6 p 4 d)$ & -80.62 & 412.47 & 331.85 & $347.6^{\mathrm{c}} 330.81^{\mathrm{d}}$ & $324 \pm 1.5^{c}$ \\
\hline $\mathrm{S}(\mathrm{RKB} / 18 s 14 p 9 d 3 f)$ & -300.55 & 984.37 & 683.82 & $751.69,{ }^{\mathrm{a}} 750.0^{\mathrm{b}}$ & \\
\hline$(\mathrm{UKB} / 17 s 9 p 6 d 1 f)$ & -286.84 & 1042.37 & 755.53 & $737.26,^{\mathrm{d}} 748.37^{\mathrm{e}}$ & $726 \pm 12^{c}$ \\
\hline $\mathrm{Se}(\mathrm{RKB} / 23 s 16 p 11 d 3 f)$ & -382.56 & 2545.98 & 2163.42 & $2203.84,{ }^{\mathrm{a}} 2422.0^{\mathrm{b}}$ & \\
\hline$(\mathrm{UKB} / 16 s 12 p 9 d 2 f)$ & -398.61 & 2829.33 & 2430.72 & $2766.7,^{\mathrm{c}} 2406.18^{\mathrm{d}}$ & $2414^{\mathrm{f}}$ \\
\hline $\mathrm{Te}(\mathrm{RKB} / 27 s 21 p 15 d 4 f)$ & -82.83 & 4261.24 & 4178.41 & $3676.86,{ }^{\mathrm{a}} 4769.4^{\mathrm{b}}$ & \\
\hline$(\mathrm{UKB} / 23 s 15 p 12 d 3 f)$ & 29.40 & 4794.37 & 4823.77 & $5694.8,{ }^{\mathrm{a}} 4569.37^{\mathrm{d}}$ & $4954^{\mathrm{g}}$ \\
\hline Po $(\mathrm{RKB} / 27 s 21 p 15 d 5 f)$ & 6080.47 & 7287.51 & 13367.98 & & \\
\hline$(\mathrm{UKB} / 24 s 19 p 13 d 5 f)$ & 6026.76 & 8320.24 & 14347.00 & $15185.7,^{\mathrm{c}} 11086.72^{\mathrm{d}}$ & \\
\hline \multicolumn{6}{|c|}{$\mathrm{XH}_{3}$} \\
\hline $\mathrm{N}(\mathrm{RKB} / 15 s 9 p 8 d 1 f)$ & -88.27 & 353.25 & 264.98 & & \\
\hline$(\mathrm{UKB} / 14 s 6 p 4 d)$ & -86.61 & 353.03 & 266.42 & & $264.5 \pm 0.05^{\mathrm{h}}$ \\
\hline $\mathrm{P}(\mathrm{RKB} / 18 s 14 p 9 d 3 f)$ & -365.02 & 911.92 & 546.90 & & \\
\hline$(\mathrm{UKB} / 17 s 9 p 6 d 1 f)$ & -345.20 & 962.13 & 616.93 & $615.02,{ }^{\mathrm{e}} 669.09^{\mathrm{i}}$ & $599.93^{\mathrm{j}}$ \\
\hline As $\mathrm{RKB} /(21 s 16 p 11 d 3 f)$ & -515.58 & 2484.67 & 1969.09 & & \\
\hline$(\mathrm{UKB} / 19 s 16 p 11 d 3 f)$ & -511.76 & 2714.34 & 2202.58 & $2457.82^{\mathrm{i}}$ & \\
\hline $\mathrm{Sb}(\mathrm{RKB} / 24 s 22 p 15 d 6 f)$ & -259.97 & 4368.36 & 4108.39 & & \\
\hline$(\mathrm{UKB} / 22 s 19 p 13 d 5 f)$ & -238.27 & 4678.15 & 4439.88 & $5077.48^{\mathrm{i}}$ & \\
\hline $\mathrm{Bi}(\mathrm{RKB} / 26 s 21 p 15 d 5 f)$ & 4406.31 & 7492.52 & 11898.83 & & \\
\hline$(\mathrm{UKB} / 23 s 19 p 13 d 4 f)$ & 4237.37 & 8337.54 & 12574.91 & $13500.86^{\mathrm{i}}$ & \\
\hline
\end{tabular}

${ }^{\mathrm{a}}$ Nonrelativistic calculations with spin-orbit corrections taken from Ref. 26.

${ }^{\mathrm{b}}$ Quasirelativistic results from a Douglas-Kroll-Hess (DKH) calculation (Ref. 27).

${ }^{\mathrm{c}}$ Calculations with complete active space (CAS) correlated method and experimental values taken from Ref. 28. ${ }^{\mathrm{d}}$ DHF calculations (nondiamagnetic approximation) taken from Ref. 29.

${ }^{\mathrm{e}}$ Results taken from Ref. 25 . They were obtained with equilibrium geometry at CCSD(T)/cc-pVTZ level plus relativistic correction calculated with DIRAC code and cc-pCVTZ basis set.

${ }^{\mathrm{f}}$ Experimental values taken from Ref. 30.

${ }^{\mathrm{g}}$ Estimated value taken from the relation reported in Ref. 31

${ }^{h}$ Estimated values derived from the spin-rotation tensor for the $\mathrm{NH}_{3}$ molecule in its ground vibrational state taken from Ref. 32

${ }^{\mathrm{i}}$ Calculations with CAS correlated method taken from Ref. 12. They are a sum of NR and semirelativistic corrections.

${ }^{\mathrm{j}}$ Experimental value with rovibrational corrections to the spin-rotation tensor from Ref. 33 .

output of calculations are exactly the same. It means that $\sigma^{d}$ does not depend on electron correlation, and relativistic effects do not modify this independence.

Following the same line of reasoning, we want to address here the question of whether relativity enhances electron correlation effects on $\sigma^{p}$. We do it from the analysis of calculations at PZOA and RPA levels, and do it within both relativistic and nonrelativistic domains. If $\Delta \sigma^{p}=\sigma^{p}$ (RPA) $-\sigma^{p}$ (PZOA) depends on whether calculations are done in the relativistic or nonrelativistic domain, this would mean that electron correlation is not independent of relativity.

In the last column of Table II, $\Delta \sigma^{p}$ is given for all studied model compounds. We can consider $\Delta \sigma^{p}$ as a measure of correlation effects. So $\left(\Delta \sigma^{p, \mathrm{R}}-\Delta \sigma^{p, \mathrm{NR}}\right) / \Delta \sigma^{p, \mathrm{NR}}$ would give us an estimate on how much relativity influences correlation effects. For Te, Sn, Po, and $\mathrm{Pb}$, we get $6.63 \%,-5.67 \%$, $-12.19 \%$, and $-28.61 \%$, respectively.

\section{B. Contributions to $\sigma^{d}$ from a closed interval of excitation energies}

As shown in Eqs. (11) and (13), the principal propagator at PZOA level of approach can be expressed as a series. The convergency of that series is restricted to virtual negativeenergy electronic states $\varepsilon_{\bar{s}}$ that fulfill the condition $2 m c^{2}$ $\leqq\left(\varepsilon_{i}-\varepsilon_{\bar{s}}\right)<4 m c^{2}$, where $\varepsilon_{i}$ is the energy of the $i$ th occupied MO. In a previous work, a large contribution of that interval of energies to $\sigma^{d}$ was found. Then, in what follows, we show an analysis on whether (i) that behavior is universal (or not), (ii) that is a function of the basis sets, and (iii) what is the total amount of its contribution to $\sigma^{d}$.

\section{Percentage of contributions from first to all orders in the series}

When calculations are done with the series of Eq. (13) truncated at first order $(n=1)$, they are equivalent to that of the LR-ESC formalism. ${ }^{12,13}$ In Table III, calculations at PZOA level of approach are given and compared with those of the series. Series results amount to more than $99 \%$ of PZOA results. In the case of lighter central atoms, the contribution is almost $100 \%$. All results of the converged series $(n=\infty)$ are closer to PZOA when compared with those of the series at first order $(n=1)$. These results are independent of the molecular model analyzed: rare gases, $\mathrm{XH}, \mathrm{XH}_{2}$, or $\mathrm{XH}_{3}$. 
TABLE II. Relativistic (nonrelativistic between parentheses) para- and diamagnetic contributions to $\sigma(\mathrm{X})$ at RPA and PZOA levels of approach. $\left[\Delta \sigma^{p}\right.$ means $\sigma^{p}(\mathrm{RPA})-\sigma^{p}(\mathrm{PZOA})$ or first-order electron correlation effects.] Results are given in ppm.

\begin{tabular}{|c|c|c|c|c|c|c|}
\hline \multirow[b]{2}{*}{$\mathrm{X}$ (basis set) } & \multicolumn{3}{|c|}{ RPA } & \multicolumn{2}{|c|}{ PZOA } & \multirow[b]{2}{*}{$\Delta \sigma^{p}$} \\
\hline & $\sigma^{p}$ & $\sigma^{d}$ & $\sigma^{\text {Total }}$ & $\sigma^{p}$ & $\sigma^{d}$ & \\
\hline \multicolumn{7}{|c|}{$\mathrm{XH}_{2}$} \\
\hline $\mathrm{O}(15 s 10 p 6 d 1 f)$ & -82.18 & 388.38 & 306.20 & -32.35 & 388.42 & -49.83 \\
\hline $\mathrm{S}(18 s 14 p 9 d 3 f)$ & -300.55 & 984.37 & 683.82 & -92.31 & 984.55 & -208.24 \\
\hline Se $(23 s 16 p 11 d 3 f)$ & -382.56 & 2545.98 & 2163.42 & 93.03 & 2546.73 & -289.53 \\
\hline $\mathrm{Te}(27 s 21 p 15 d 4 f)$ & $\begin{array}{c}-82.83 \\
(-1649.27)\end{array}$ & $\begin{array}{c}4261.24 \\
(4824.03)\end{array}$ & $\begin{array}{c}4178.41 \\
(3174.76)\end{array}$ & $\begin{array}{c}975.22 \\
(-657.02)\end{array}$ & $\begin{array}{c}4262.72 \\
(4824.04)\end{array}$ & $\begin{array}{c}-1058.05 \\
(-992.25)\end{array}$ \\
\hline Po $(27 s 21 p 15 d 5 f 1 g)$ & $\begin{array}{c}6090.86 \\
(-3013.08)\end{array}$ & $\begin{array}{c}7297.23 \\
(9093.39)\end{array}$ & $\begin{array}{r}13388.86 \\
(6080.31)\end{array}$ & $\begin{array}{c}7752.95 \\
(-1120.23)\end{array}$ & $\begin{array}{c}7299.98 \\
(9093.39)\end{array}$ & $\begin{array}{c}-1662.09 \\
(-1892.85)\end{array}$ \\
\hline \multicolumn{7}{|c|}{$\mathrm{XH}_{3}$} \\
\hline $\mathrm{N}(15 s 10 p 6 d 1 f)$ & -88.41 & 336.18 & 247.77 & -43.11 & 336.20 & -45.30 \\
\hline $\mathrm{P}(18 s 14 p 9 d 3 f)$ & -365.02 & 911.92 & 546.90 & -140.55 & 912.07 & -224.47 \\
\hline As $(\mathrm{RKB} /(21 s 16 p 11 d 3 f)$ & -515.58 & 2484.67 & 1969.09 & -24.61 & 2485.41 & -490.97 \\
\hline $\mathrm{Sb}(24 s 22 p 15 d 6 f)$ & -259.97 & 4368.36 & 4108.39 & 700.78 & 4369.86 & -960.75 \\
\hline $\mathrm{Bi}(26 s 21 p 15 d 5 f)$ & 4406.31 & 7492.52 & 11898.83 & 6495.74 & 7495.62 & -2089.43 \\
\hline \multicolumn{7}{|c|}{$\mathrm{XH}_{4}$} \\
\hline $\operatorname{Sn}(21 s 19 p 15 d 5 f)$ & $\begin{array}{c}-402.97 \\
(-1819.52)\end{array}$ & $\begin{array}{c}4076.57 \\
(4576.34)\end{array}$ & $\begin{array}{r}3673.60^{\mathrm{a}} \\
(2756.82)\end{array}$ & $\begin{array}{c}468.10 \\
(-896.11)\end{array}$ & $\begin{array}{c}4077.89 \\
(4576.35)\end{array}$ & $\begin{array}{c}-871.07 \\
(-923.41)\end{array}$ \\
\hline $\mathrm{Pb}(27 s 22 p 17 d 12 f 3 g)$ & $\begin{array}{c}4955.12 \\
(-3168.55)\end{array}$ & $\begin{array}{c}7835.03 \\
(9558.22)\end{array}$ & $\begin{array}{r}12790.15^{\mathrm{b}} \\
(6389.67)\end{array}$ & $\begin{array}{c}6095.82 \\
(-1570.63)\end{array}$ & $\begin{array}{c}7838.44 \\
(9558.22)\end{array}$ & $\begin{array}{c}-1140.70 \\
(-1597.92)\end{array}$ \\
\hline \multicolumn{7}{|c|}{$\mathrm{SnXH}_{3}$} \\
\hline $\operatorname{Sn}(24 s 22 p 15 d 5 f)$ & -758.37 & 4205.68 & 3447.31 & 279.18 & 4206.98 & -1037.55 \\
\hline $\mathrm{Br}(18 s 13 p 8 d 2 f)$ & 559.84 & 2277.93 & 2837.77 & 638.14 & 2278.61 & -78.30 \\
\hline $\operatorname{Sn}(21 s 19 p 15 d 5 f)$ & -719.78 & 4261.42 & 3541.64 & 279.18 & 4262.73 & -998.96 \\
\hline I $(26 s 21 p 18 d 9 f 1 g)$ & 1838.70 & 4728.38 & 6567.08 & 2122.77 & 4730.43 & -284.07 \\
\hline
\end{tabular}

${ }^{\mathrm{a}}$ Relativistic CAS self-consistent field (SCF) results: $4017.54 \mathrm{ppm}$ (3972.23 ppm), taken from Ref. 34.

${ }^{\mathrm{b}}$ Relativistic CAS (SCF) results: $10091.51 \mathrm{ppm}$ (10 $\left.004.06 \mathrm{ppm}\right)$, taken from Ref. 34.

A similar analysis was done for two different $\mathrm{XH}_{4}(\mathrm{X}$ $=\mathrm{Sn}$ and $\mathrm{Pb}$ ) molecular models and also for $\mathrm{SnBrH}_{3}$, in which there are two equivalent heavy atoms. When one $\mathrm{H}$ atom is replaced by $\mathrm{Br}$, the percentage of contributions to the diamagnetic term remains almost the same, i.e., close to $100 \%$.

Results of calculations by the series are less closer to PZOA results when $Z$ becomes larger. This seems to be due to the lack of large enough basis set for heavier atoms. In order to analyze whether this conjecture is or is not right, we studied three molecular systems, improving the basis set of their central atoms. Selected model compounds were $\mathrm{SnH}_{4}$, $\mathrm{TeH}_{2}$, and HI. Results of RPA, PZOA, and first order and converged series calculations are given in Table IV. There it is shown that when the basis set was highly improved, RPA and PZOA results still maintain a similar difference between them. On the other hand, the difference between calculations at PZOA level and the converged series becomes closer. This behavior is more pronounced for Te due to the fact that a larger basis set is used for it. In the case of the largest basis set used, i.e., $(27 s 21 p 15 d 4 f)$, the calculations at PZOA level and the converged series results differ by $0.59 \%$. In the same vein, results from the first-order series calculations become less closer to PZOA when a more complete basis set is ap- plied, which means that one should exercise great care in using a saturated basis set when applying LR-ESC or equivalent methods.

We also studied the dependence of the percentage of negative-energy electronic states that have energies below $-4 m c^{2}$ on the size of the basis set. For HI, we found out that the percentage grows when the size of basis set is increased as follows: $5 \%$ for $(17 s 14 p 10 d 3 f), 9 \%$ for $(20 s 16 p 13 d 4 f)$, and $15 \%$ for $(25 s 21 p 19 d 8 f)$. At the same time, as shown in Table IV, when the basis set is improved, the percentage of the total contribution of excitations belonging to the interval referred to above does increase. For basis set $(17 s 14 p 10 d 3 f)$, the total contribution amounts to $100.88 \%$, and for basis set $(25 s 21 p 19 d 8 f)$, the total contribution of the interval is $100.63 \%$ compared with PZOA calculations.

Then by improving the description of all electronic states, a larger percentage of them will have energies lower than $-4 m c^{2}$. This means that the negative-energy branch is better described as a whole; in particular, the negative-energy states with energies higher than $-4 m c^{2}$. At the same time, the contribution from excitation energies belonging to the interval of interest becomes closer to $100 \%$. In the limit of a complete basis set, the total energy spectra will be described with the highest accuracy, and the percentage of the contri- 
TABLE III. Comparison of relativistic diamagnetic contributions at PZOA level of approach and two different levels of its series development.

\begin{tabular}{|c|c|c|c|c|c|c|c|}
\hline \multirow[b]{2}{*}{ X (basis set) } & \multirow{2}{*}{$\begin{array}{c}\mathrm{PZOA} \\
\sigma^{d}\end{array}$} & \multicolumn{2}{|c|}{ Series } & \multirow[b]{2}{*}{$\mathrm{X}$ (basis set) } & \multirow{2}{*}{$\begin{array}{c}\mathrm{PZOA} \\
\sigma^{d}\end{array}$} & \multicolumn{2}{|c|}{ Series } \\
\hline & & $\sigma^{d}(n=1)$ & $\sigma^{d}(n=\infty)$ & & & $\sigma^{d}(n=1)$ & $\sigma^{d}(n=\infty)$ \\
\hline \multicolumn{4}{|c|}{$\mathrm{X}=$ Rare gases } & \multicolumn{4}{|c|}{$\mathrm{XH}$} \\
\hline $\operatorname{Kr}(19 s 13 p 17 d 12 f)$ & 2945.70 & $\begin{array}{l}2921.19 \\
\quad(99.17 \%)\end{array}$ & $\begin{array}{l}2965.35 \\
(100.67 \%)\end{array}$ & $\operatorname{Br}(22 s 18 p 20 d 5 f)$ & 2679.97 & $\begin{array}{l}2669.33 \\
(99.60 \%)\end{array}$ & $\begin{array}{l}2693.65 \\
(100.51 \%)\end{array}$ \\
\hline $\mathrm{Xe}(23 s 19 p 21 d 10 f)$ & 4770.05 & $\begin{array}{l}4757.18 \\
\quad(99.73 \%)\end{array}$ & $\begin{array}{l}4806.10 \\
(100.76 \%)\end{array}$ & I $(25 s 21 p 19 d 8 f)$ & 4646.03 & $\begin{array}{l}4625.05 \\
(99.55 \%)\end{array}$ & $\begin{array}{l}4675.30 \\
(100.63 \%)\end{array}$ \\
\hline $\operatorname{Rn}(24 s 18 p 13 d 8 f)$ & 8173.51 & $\begin{array}{l}7997.76 \\
(97.85 \%)\end{array}$ & $\begin{array}{l}8222.75 \\
(100.60 \%)\end{array}$ & At $(22 s 17 p 13 d 8 f)$ & 8106.96 & $\begin{array}{l}7924.75 \\
(97.75 \%)\end{array}$ & $\begin{array}{l}8173.52 \\
(100.82 \%)\end{array}$ \\
\hline \multicolumn{4}{|c|}{$\mathrm{XH}_{2}$} & \multicolumn{4}{|c|}{$\mathrm{XH}_{3}$} \\
\hline $\mathrm{O}(15 s 10 p 6 d 1 f)$ & 388.42 & $\begin{array}{l}388.36 \\
(99.98 \%)\end{array}$ & $\begin{array}{l}388.44 \\
(100.01 \%)\end{array}$ & $\mathrm{N}(15 s 9 p 8 d 1 f)$ & 353.44 & $\begin{array}{l}353.41 \\
(99.99 \%)\end{array}$ & $\begin{array}{l}353.45 \\
(100.00 \%)\end{array}$ \\
\hline $\mathrm{S}(18 s 14 p 9 d 3 f)$ & 984.55 & $\begin{array}{l}983.76 \\
(99.92 \%)\end{array}$ & $\begin{array}{l}984.87 \\
(100.03 \%)\end{array}$ & $\mathrm{P}(18 s 14 p 9 d 3 f)$ & 912.07 & $\begin{array}{l}911.40 \\
(99.93 \%)\end{array}$ & $\begin{array}{l}912.28 \\
(100.02 \%)\end{array}$ \\
\hline $\operatorname{Se}(23 s 16 p 11 d 3 f)$ & 2546.73 & $\begin{array}{l}2541.50 \\
(99.79 \%)\end{array}$ & $\begin{array}{l}2555.34 \\
(100.34 \%)\end{array}$ & As $(21 s 16 p 11 d 3 f)$ & 2485.42 & $\begin{array}{l}2464.75 \\
\quad(99.16 \%)\end{array}$ & $\begin{array}{l}2489.18 \\
(100.15 \%)\end{array}$ \\
\hline $\mathrm{Te}(27 s 21 p 15 d 4 f)$ & 4262.72 & $\begin{array}{l}4203.73 \\
(98.62 \%)\end{array}$ & $\begin{array}{l}4288.01 \\
(100.59 \%)\end{array}$ & $\mathrm{Sb}(24 s 22 p 15 d 6 f)$ & 4369.86 & $\begin{array}{l}4316.26 \\
(98.77 \%)\end{array}$ & $\begin{array}{l}4395.96 \\
(100.60 \%)\end{array}$ \\
\hline Po $(27 s 21 p 15 d 5 f)$ & 7290.26 & $\begin{array}{l}7155.39 \\
(98.15 \%)\end{array}$ & $\begin{array}{l}7337.65 \\
(100.65 \%)\end{array}$ & $\mathrm{Bi}(26 s 21 p 15 d 5 f)$ & 7495.62 & $\begin{array}{l}7391.43 \\
\quad(98.61 \%)\end{array}$ & $\begin{array}{l}7552.59 \\
(100.76 \%)\end{array}$ \\
\hline \multicolumn{4}{|c|}{$\mathrm{XH}_{4}$} & \multicolumn{4}{|c|}{$\mathrm{SnBrH}_{3}$} \\
\hline Sn $(21 s 19 p 15 d 5 f)$ & 4077.89 & $\begin{array}{l}4067.20 \\
\quad(99.74 \%)\end{array}$ & $\begin{array}{l}4108.60 \\
(100.76 \%)\end{array}$ & $\operatorname{Sn}(24 s 22 p 15 d 5 f)$ & 4206.98 & $\begin{array}{l}4203.95 \\
\quad(99.93 \%)\end{array}$ & $\begin{array}{l}4241.74 \\
(100.83 \%)\end{array}$ \\
\hline $\mathrm{Pb}(27 s 22 p 17 d 12 f 3 g)$ & 7838.44 & $\begin{array}{l}7633.43 \\
(97.38 \%)\end{array}$ & $\begin{array}{l}7911.55 \\
(100.93 \%)\end{array}$ & $\operatorname{Br}(18 s 13 p 8 d 2 f)$ & 2278.61 & $\begin{array}{l}2267.76 \\
(99.52 \%)\end{array}$ & $\begin{array}{l}2284.37 \\
(100.25 \%)\end{array}$ \\
\hline
\end{tabular}

bution of the energy interval to $\sigma^{d}$ should be exactly $100 \%$. This is a proof that the energy interval is not a basis set artifact.

From these analyses, it seems clear that the above mentioned interval of excitation energies gives the total contribution to $\sigma^{d}$ independent of the type and geometry of all compounds studied here. When each geometry, bond length, and bond angle was modified, the pattern of results for the same calculations was always the same.

\section{Pattern of contributions to $\sigma^{d}(X)$ for $X$ belonging to the $\mathrm{XH}_{2}$ model compounds}

In Fig. 1, the contribution from the complete negativeenergy branch of energies to $\sigma^{d}$ for the series of $\mathrm{XH}_{2}$ type of compounds at PZOA level is shown. There are some contributions of the heavier elements in the region of energies smaller than $-3 m c^{2}$. This behavior is similar to that of rare gases and hydrogen halides. ${ }^{4}$ It could explain why the LRESC is not good enough for elements of the sixth row in the Periodic Table. In such a case, one should include more than two terms in the series of Eq. (13) if one wants to seek accurate results.

The expanded pattern of contributions given in Fig. 2 shows that for lighter elements, all contributions arise from virtual states close to $-2 m c^{2}$. Then the series of Eq. (13) converges faster to PZOA results for those elements compared with convergence of the heaviest elements. Only for Te and Po, there are few important contributions $(>50 \mathrm{ppm})$ from virtual states with energies lower than $-3 m c^{2}$.

\section{Pattern of $1 s_{1 / 2}$ contributions}

Another important point to be clarified is related to $1 s_{1 / 2}$ contributions to $\sigma^{d}$. As observed in Fig. 3, the deepest occupied molecular orbital is the most important. When comparing both figures, one realizes that in the case of Te both the total and $1 s_{1 / 2}$ contributions follow quantitatively the same pattern for contributions of states, with energies a little below $-2 m c^{2}$. This behavior is more pronounced in the case of Po. Contributions which include negative-energy one-electron states close to $-2 m c^{2}$ are originated in some other different occupied molecular states, none of them being the most relevant.

\section{CONCLUDING REMARKS}

Following some of our previous studies related with the electronic origin of relativistic effects in nuclear magnetic molecular properties within full relativistic quantum theory, we present here two important new results.

The analysis of the influence of relativity on electron correlation effects is not an easy task. There are not many theoretical tools that could give at least preliminary results on that matter with confidence. The relativistic polarization propagator is quite a good candidate because its formal definition is exactly the same within both relativistic and nonrelativistic domains. Starting from full relativistic expressions, one can get their nonrelativistic limit, by theory or by numerical calculations, making $c$ approach infinity.

We have made accurate calculations at RPA and PZOA levels of approach within relativistic and nonrelativistic do- 
TABLE IV. Percentage of contributions of the series with respect to PZOA calculations depending on the completeness of the basis set.

\begin{tabular}{|c|c|c|c|c|}
\hline \multirow[b]{2}{*}{ X (base) } & \multirow{2}{*}{$\begin{array}{l}\mathrm{RPA} \\
\sigma^{d}\end{array}$} & \multirow{2}{*}{$\begin{array}{c}\text { PZOA } \\
\sigma^{d}\end{array}$} & \multicolumn{2}{|c|}{ Series } \\
\hline & & & $\sigma^{d}(n=1)$ & $\sigma^{d}(n=\infty)$ \\
\hline \multicolumn{5}{|c|}{$\mathrm{SnH}_{4}$} \\
\hline $\operatorname{Sn}(18 s 15 p 10 d 2 f)$ & 3611.16 & 3612.16 & $\begin{array}{l}3603.24 \\
\quad(99.75 \%)\end{array}$ & $\begin{array}{l}3644.32 \\
(100.89 \%)\end{array}$ \\
\hline $\operatorname{Sn}(19 s 15 p 12 d 4 f)$ & 3917.64 & 3918.80 & $\begin{array}{l}3915.69 \\
(99.92 \%)\end{array}$ & $\begin{array}{l}3951.95 \\
(100.85 \%)\end{array}$ \\
\hline $\operatorname{Sn}(21 s 17 p 13 d 5 f)$ & 3911.00 & 3912.15 & $\begin{array}{l}3906.82 \\
\quad(99.86 \%)\end{array}$ & $\begin{array}{l}3942.86 \\
(100.78 \%)\end{array}$ \\
\hline $\operatorname{Sn}(21 s 19 p 15 d 5 f)$ & 4076.57 & 4077.89 & $\begin{array}{l}4067.20 \\
(99.74 \%)\end{array}$ & $\begin{array}{l}4108.60 \\
(100.76 \%)\end{array}$ \\
\hline \multicolumn{5}{|c|}{$\mathrm{TeH}_{2}$} \\
\hline $\mathrm{Te}(21 s 16 p 10 d 1 f)$ & 4142.43 & 4143.82 & $\begin{array}{l}4106.87 \\
(99.11 \%)\end{array}$ & $\begin{array}{l}4191.71 \\
(101.16 \%)\end{array}$ \\
\hline $\mathrm{Te}(23 s 17 p 12 d 2 f)$ & 4224.11 & 4225.59 & $\begin{array}{l}4185.04 \\
\quad(99.04 \%)\end{array}$ & $\begin{array}{l}4253.57 \\
(100.66 \%)\end{array}$ \\
\hline $\mathrm{Te}(25 s 19 p 13 d 3 f)$ & 4231.57 & 4233.09 & $\begin{array}{l}4191.77 \\
\quad(99.02 \%)\end{array}$ & $\begin{array}{l}4259.32 \\
(100.62 \%)\end{array}$ \\
\hline $\mathrm{Te}(27 s 21 p 15 d 4 f)$ & 4261.24 & 4262.72 & $\begin{array}{l}4203.73 \\
(98.62 \%)\end{array}$ & $\begin{array}{l}4288.01 \\
(100.59 \%)\end{array}$ \\
\hline \multicolumn{5}{|c|}{$\mathrm{IH}$} \\
\hline I $(17 s 14 p 10 d 3 f)$ & 4155.86 & 4157.35 & $\begin{array}{l}4140.25 \\
\quad(99.59 \%)\end{array}$ & $\begin{array}{l}4193.93 \\
(100.88 \%)\end{array}$ \\
\hline $\mathrm{I}(18 s 15 p 12 d 4 f)$ & 4136.80 & 4138.28 & $\begin{array}{l}4130.17 \\
\quad(99.80 \%)\end{array}$ & $\begin{array}{l}4173.46 \\
(100.85 \%)\end{array}$ \\
\hline $\mathrm{I}(20 s 16 p 13 d 4 f)$ & 4127.89 & 4129.37 & $\begin{array}{l}4118.08 \\
(99.73 \%)\end{array}$ & $\begin{array}{l}4162.60 \\
(100.80 \%)\end{array}$ \\
\hline $\mathrm{I}(21 s 18 p 15 d 5 f)$ & 4430.67 & 4432.43 & $\begin{array}{l}4390.11 \\
\quad(99.05 \%)\end{array}$ & $\begin{array}{l}4463.52 \\
(100.70 \%)\end{array}$ \\
\hline $\mathrm{I}(25 s 21 p 19 d 8 f)$ & 4644.18 & 4646.03 & $\begin{array}{l}4625.05 \\
\quad(99.55 \%)\end{array}$ & $\begin{array}{l}4675.30 \\
(100.63 \%)\end{array}$ \\
\hline
\end{tabular}

mains. The model compounds studied here are noble gases, $\mathrm{XH}(\mathrm{X}=\mathrm{Br}, \mathrm{I}$, and $\mathrm{At}), \mathrm{XH}_{2}(\mathrm{X}=\mathrm{O}, \mathrm{S}, \mathrm{Se}, \mathrm{Te}$, and $\mathrm{Po}), \mathrm{XH}_{3}$ $(\mathrm{X}=\mathrm{N}, \mathrm{P}, \mathrm{As}, \mathrm{Sb}$, and $\mathrm{Bi}), \mathrm{XH}_{4}(\mathrm{X}=\mathrm{Sn}$ and $\mathrm{Pb})$, and $\mathrm{SnXH}_{3}$ $(\mathrm{X}=\mathrm{Br}$ and $\mathrm{I})$. For all those compounds, we consider that the behavior of $\sigma$ should be similar within both domains. Then

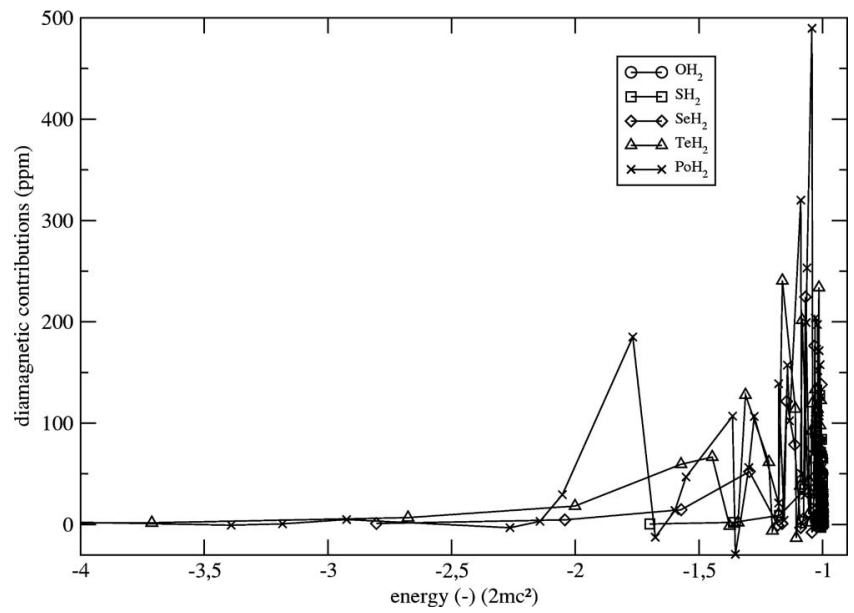

FIG. 1. Pattern of negative-energy states contributing to $\sigma^{d}$ of $\mathrm{XH}_{2}$ model compounds.

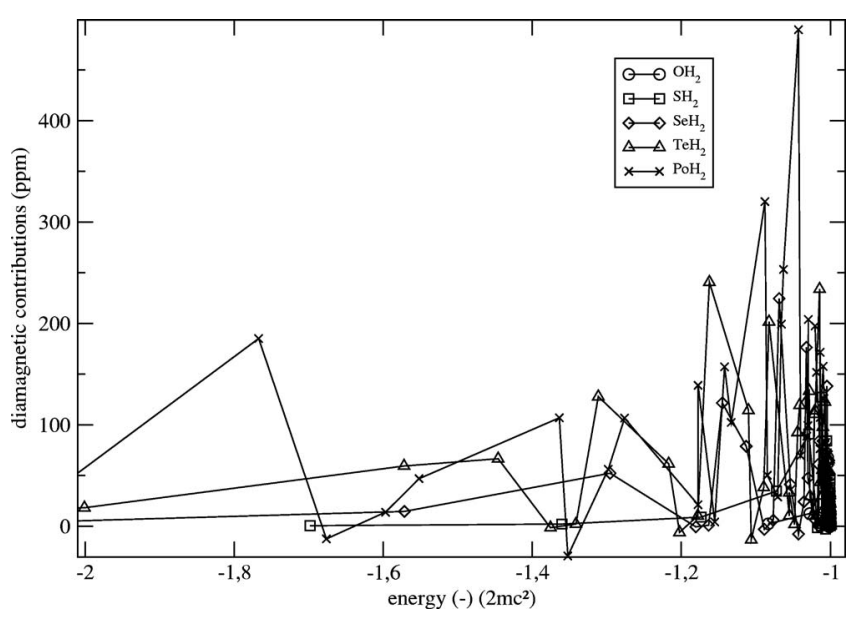

FIG. 2. Extended pattern of negative-energy states contributing to $\sigma^{d}$ of $\mathrm{XH}_{2}$ model compounds.

RPA results will include a large amount of the whole electron correlation that must be included in the calculation of that magnetic property.

We consider that $\Delta \sigma=\sigma^{\mathrm{RPA}}(\mathrm{X})-\sigma^{\mathrm{PZOA}}(\mathrm{X})$ is a measure of the correlation effects on the nuclear magnetic shielding of $\mathrm{X}$ atom. The analysis of $\Delta \sigma^{d}$ for all model compounds shows that the diamagnetic contribution does not depend on electron correlation in both relativistic and nonrelativistic domains. This finding is quite clear and completely general. On the other hand, $\left(\Delta \sigma^{p, \mathrm{R}}-\Delta \sigma^{p, \mathrm{NR}}\right) / \Delta \sigma^{p, \mathrm{NR}}$ would give us an estimate on how much relativity enhances correlation effects for paramagnetic contributions. As discussed and shown in Sec. IV A, relativity modifies correlation effects for paramagnetic contributions. For atoms belonging to the fifth row of the Periodic Table, the influence is smaller than 7\%, but for atoms belonging to the next row, the influence is more important: $\sim 12 \%$ for $\mathrm{Po}$ in $\mathrm{PoH}_{2}$ and $\sim 29 \%$ for $\mathrm{Pb}$ in $\mathrm{PbH}_{4}$.

The second novelty we present here is related to the amount of the contribution that a definite interval of excitation energies (which we discovered recently) gives to the diamagnetic term of $\sigma$. We show now that the interval $2 m c^{2} \leqq\left(\varepsilon_{i}-\varepsilon_{\bar{S}}\right)<4 m c^{2}$ gives the total contribution to $\sigma^{d}$, and

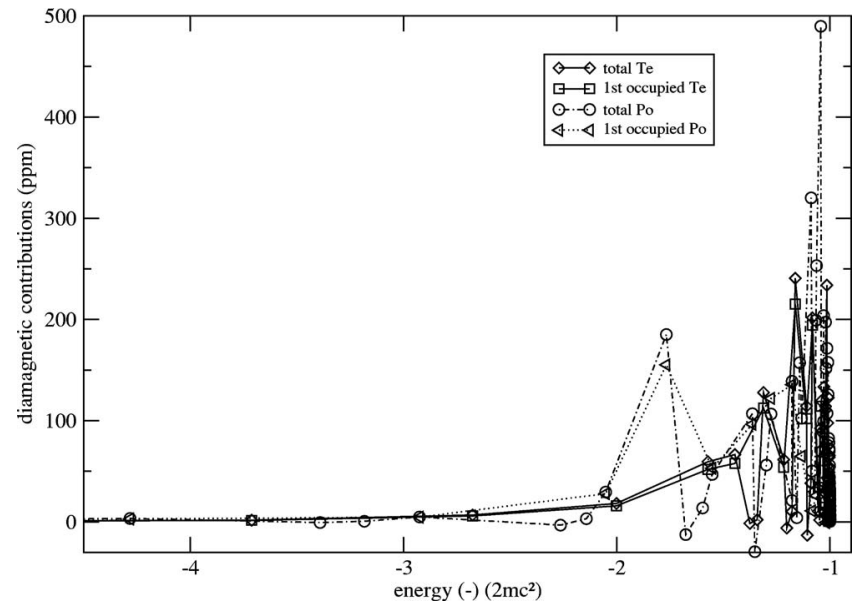

FIG. 3. Comparison of negative-energy contributions to $\sigma^{d}(\mathrm{Te})$ and $\sigma^{d}(\mathrm{Po})$ considering excitations from all occupied relativistic MOs vs the first occupied relativistic MO. 
this is independent of the model compound studied and also on the basis set used. In a previous work, we have shown that the amount of contribution was close to $100 \%$, like $98 \%$ for heaviest elements.

Analyzing the pattern of such contributions as a function of the atomic number of the elements, it is observed that when $Z$ goes down in the Periodic Table, there arise some excitations in the region close to the lower limit that contribute significantly. This can explain why the LR-ESC formalism has some difficulties in reproducing accurately the shielding of heavy atoms that belong to the sixth row. In such a case, it would be necessary to include more terms in the series development for the LR-ESC formalism in order to get results closer to full relativistic linear response theoretical calculations.

We also found that the $1 s_{1 / 2}$ pattern of contributions does not match the whole pattern of the total contributions. They match each other for the deepest part of the interval of energies. Contributions from other deep lying occupied states are relevant in the first part of the interval.

Finally, when trying to grasp an understanding of the origin of the new rule that fixes an interval for the excitations to negative-energy electronic states contributing to $\sigma^{d}$, one should look at the theory based on QED for describing magnetic molecular properties. Progress in this direction is under current work in our laboratory.

\section{ACKNOWLEDGMENTS}

We gratefully acknowledge partial support from SGCyTUNNE, the Argentinian National Research Council for Science and Technology (CONICET, Grant No. PIP 5119/2004), and the Argentinian Agency for Promotion of Science and Technology (FONCYT, Grant No. PICT 21604/2004).

We also thank Dr. Sergio Gomez for his assistance in the series calculations.

\footnotetext{
${ }^{1}$ G. A. Aucar, T. Saue, L. Visscher, and H. J. Aa. Jensen, J. Chem. Phys. 110, 6208 (1999)

${ }^{2}$ R. Szmytkowski, Phys. Rev. A 65, 32112 (2002).

${ }^{3}$ W. Kutzelnigg, Phys. Rev. A 67, 32109 (2003).

${ }^{4}$ S. S. Gómez, A. Maldonado, and G. A. Aucar, J. Chem. Phys. 123, 214108 (2005).

${ }^{5}$ Y. Xiao, D. Peng, and W. Liu, J. Chem. Phys. 126, 81101 (2007).
}

${ }^{6}$ R. H. Romero and G. A. Aucar, Int. J. Mol. Sci. 3, 914 (2002).

${ }^{7}$ R. H. Romero and G. A. Aucar, Phys. Rev. A 65, 53411 (2002).

${ }^{8}$ M. M. Sternheim, Phys. Rev. 128, 676 (1962).

${ }^{9}$ P. Pyykkö, Chem. Phys. 22, 289 (1977); 74, 1 (1983).

${ }^{10}$ G. A. Aucar and J. Oddershede, Int. J. Quantum Chem. 47, 425 (1993).

${ }^{11}$ P. F. Provasi, C. A. Gómez, and G. A. Aucar, J. Phys. Chem. A 108, 6231 (2004).

${ }^{12}$ J. I. Melo, M. C. Ruiz de Azúa, C. G. Giribet, G. A. Aucar, and R. H. Romero, J. Chem. Phys. 118, 104 (2003).

${ }^{13}$ J. I. Melo, M. C. Ruiz de Azúa, C. G. Giribet, G. A. Aucar, and P. F. Provasi, J. Chem. Phys. 121, 6798 (2004).

${ }^{14}$ S. S. Gómez, J. I. Melo, R. H. Romero, G. A. Aucar, and M. C. Ruiz de Azúa, J. Chem. Phys. 122, 64103 (2005).

${ }^{15} \mathrm{~J}$. Vaara, P. Manninen, and P. Lantto, in Calculation of NMR and EPR Parameters: Theory and Applications, edited by M. Kaupp, M. Buhl, and V. G. Malkin (Wiley-VCH, Weinheim, 2004), p. 209.

${ }^{16}$ P. Lantto, R. H. Romero, S. S. Gómez, G. A. Aucar, and J. Vaara, J. Chem. Phys. 125, 184113 (2006).

${ }^{17}$ T. Saue, V. Bakken, T. Enevoldsen, T. Helgaker, H. J. Aa. Jensen, J. K. Laerdahl, K. Ruud, J. Thyssen, and L. Visscher, DIRAC, Release 4.0, a relativistic ab initio electronic structure program, University of Southern Denmark, Odense, 2004, http://dirac.chem.sdu.dk

${ }^{18}$ Tables of Interatomic Distances and Configurations in Molecules and Ions, edited by L. E. Sutton (The Chemical Society, London, 1965).

${ }^{19}$ S. S. Gómez, R. H. Romero, and G. A. Aucar, J. Chem. Phys. 117, 7942 (2002).

${ }^{20}$ L. Visscher, Adv. Quantum Chem. 48, 369 (2005).

${ }^{21}$ A. J. Sadlej, Theor. Chim. Acta 79, 123 (1991); 81, 45 (1991); 81, 339 (1992); V. Kello and A. J. Sadlej, Theor. Chim. Acta 83, 351 (1992).

${ }^{22}$ K. Faegri, Jr. (private communication); see also http://folk.uio.no/knutf/ bases/one.

${ }^{23} \mathrm{~J}$. Oddershede, in Methods in Computational Molecular Physics, edited by G. H. F. Diercksen and S. Wilson (Reidel, Dordrecht, 1983), pp. $258-260$.

${ }^{24}$ S. P. A. Sauer, I. Paidarova, and J. Oddershede, Theor. Chim. Acta 88, 351 (1994).

${ }^{25}$ A. Antusek and M. Jaszunski, Mol. Phys. 104, 1463 (2006).

${ }^{26}$ J. Vaara, K. Ruud, and O. Vahtras, J. Chem. Phys. 111, 2900 (1999).

${ }^{27}$ M. Hada, R. Fukuda, and H. Nakatsuji, Chem. Phys. Lett. 321, 452 (2000).

${ }^{28}$ P. Manninen, P. Lantto, J. Vaara, and K. Ruud, J. Chem. Phys. 119, 2623 (2003).

${ }^{29}$ P. Manninen, K. Ruud, P. Lantto, and J. Vaara, J. Chem. Phys. 122, 114107 (2005); 124, 149901(E) (2006).

${ }^{30}$ H. Duddeck, Prog. Nucl. Magn. Reson. Spectrosc. 27, 1 (1995).

${ }^{31}$ Y. Ruiz-Morales, G. Schreckenbach, and T. Ziegler, J. Phys. Chem. A 101, 4121 (1997)

${ }^{32}$ C. J. Jameson, A. de Dios, and A. K. Jameson, J. Chem. Phys. 95, 1069 (1991).

${ }^{33}$ C. J. Jameson, A. de Dios, and A. K. Jameson, J. Chem. Phys. 95, 9042 (1991).

${ }^{34}$ M. Jaszunski and K. Ruud, Mol. Phys. 104, 2139 (2006). 